\title{
Transport Properties of Disordered Graphene Layers
}

\author{
M. Gryglas-Borysiewicz ${ }^{a}$, B. Jounult ${ }^{a, b, c}$, J. TwOrzydŁo $^{d}$, S. LeWińska $^{a}$, \\ W. StrupińsKI ${ }^{e}$ AND J.M. BARANOWSKI ${ }^{a}$ \\ ${ }^{a}$ Institute of Experimental Physics, University of Warsaw, Hoża 69, 00-681 Warszawa, Poland \\ ${ }^{b}$ UMR 5650, CNRS, cc074, pl. Eugene Bataillon, 34095 Montpellier cedex 5, France \\ ${ }^{c}$ Groupe d'Etude des Semiconducteurs, Université Montpellier 2, cc074 \\ pl. Eugene Bataillon, 34095 Montpellier Cedex 5, France \\ ${ }^{d}$ Institute of Theoretical Physics, University of Warsaw, Hoża 69, 00-681 Warsaw, Poland \\ ${ }^{e}$ Institute of Electronic Materials Technology, Wólczyńska 133, 01-919 Warsaw, Poland
}

\begin{abstract}
Samples consisting of a few layers of graphene obtained by thermal decomposition of $\mathrm{SiC}$ were studied by means of transport experiments at $4 \mathrm{~K}$ and in a magnetic field up to $7 \mathrm{~T}$. Transport data show that the samples have a two-dimensional character. Magnetoresistance has an approximately linear character at high magnetic fields, which has been previously observed in graphite samples, and a negative magnetoresistance, at low magnetic fields. The transverse resistivity $\rho_{x y}$ is nonlinear as a function of $B$, which can be described using a many-carrier model.
\end{abstract}

PACS numbers: 73.21.Ac, 72.20.Fr, 73.43.Qt, 73.63.Bd

\section{Introduction}

For the last several years there has been a growing interest in graphene - a single layer of graphite. Its physical properties make it interesting for basic physics and promising for high speed and high power electronics. However, the development of graphene technology demands methods of large scale production. One of them is the thermal decomposition of SiC. This work shows preliminary transport measurements made on a few-layer graphene obtained with this method.

\section{Samples and experiment}

The graphene layers were obtained with thermal decomposition of $\mathrm{Si}$ atoms from a semi-insulating on-axis 4H-SiC (000-1) [1]. Two samples were investigated: \#313, having about 6 graphene sheets and \#337 with about 20 graphene sheets, as estimated by means of absorption measurements [2]. Both were $4 \times 2 \mathrm{~mm}^{2}$ large and structurally inhomogeneous with graphene flakes reaching $1.5 \mu \mathrm{m}$. Electrical ohmic contacts have been made with indium, in a Hall-bar configuration.

Transport studies have been performed at liquid helium temperature, with magnetic field ranging up to $7.5 \mathrm{~T}$. At $4.2 \mathrm{~K}$ the two samples have substantially different resistivities: $\rho \approx 16.5 \mathrm{k} \Omega$ for $\# 337$ and $\rho \approx 700 \Omega$ for $\# 313$. This is counterintuitive, because the thinner sample has the lowest resistivity. It implies either a large difference in carrier concentrations, or additional structural degradations in the thicker sample.

\section{Results and discussion}

The samples, consisting of a few layers, were expected to have two-dimensional character. In order to verify this thesis, the dependence of resistance on magnetic field direction was measured. Figure 1 shows the results obtained for one of the samples in the two configurations: field perpendicular to the plane of the layers and in the plane. In the former case, one can observe an increase of the resistance of about $70 \%$ for maximum field, whereas in the latter, the change is negligible. The qualitative difference between " $\perp$ plane" and "in-plane" configurations allows us to conclude that the observed effect was an orbital (and not a spin) one and that, indeed, the layers show a two-dimensional character.

\subsection{Magnetoresistivity}

Figure 1 shows the measured resistivity for the two samples studied. Regardless the differences in the magnitude, the magnetoresistance shows many common features for both samples. First, there is a negative magnetoresistance observed at low magnetic fields with a characteristic maximum at $B=0 \mathrm{~T}$. The half-width of the maximum is about $\approx 1 \mathrm{~T}$ for sample $\# 337$ and $\approx 0.2 \mathrm{~T}$ for sample \#313. The detailed shape of $\rho(B)$ differs when measured on different contacts for the same sample, suggesting problems with inhomogeneities or anisotropy. Therefore further studies are needed to determine if the negative magnetoresistance is due to weak localization [3], classical non-Markovian processes [4] or other 
phenomena [5]. Second, there is an almost linear dependence of resistivity on $B$ in the range 1-7 T. The relative amplitude of the magnetoresistance is much higher in sample \#313 (70\%), suggesting that this sample has the highest mobility. Such a linear trend is also observed in high oriented pyrolytic graphite (HOPG) [5]. The origin is still unclear and could be due to the disorder [6]. In the following, we neglect the disorder and we explain the behaviour of the resistivities with a multi-carrier model.

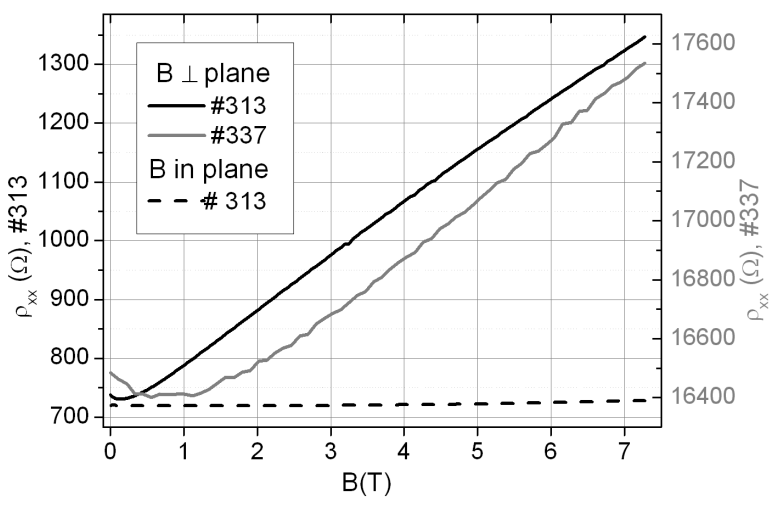

Fig. 1. The longitudinal resistances measured as a function of magnetic field $B$. Black lines: \#313 measured with $B$ perpendicular to the layer (solid line) and with $B$ parallel to the layer (dashed line). Grey solid line: \#337 with $B$ perpendicular to the layer.

\subsection{Resistivity tensor and mobility spectrum}

The measurements of a full resistivity tensor have been performed for sample \#313. Both longitudinal and transverse voltages were measured for two directions of magnetic field and current and magnetoresistance tensor was calculated (see Fig. 2a). The Hall component $\left(\rho_{x y}\right)$ is negative, indicating dominant electron conductance. A rough linear fit gives a Hall concentration $n_{\mathrm{e}}=1.5 \times 10^{13} \mathrm{~cm}^{-2}$. However, $\rho_{x y}(B)$ shows a nonlinearity. Nonlinear behaviour can in principle be explained by the presence of many conductance channels. In order to check what channels are needed to fit the data, a mobility spectrum analysis [7] was performed. Being beyond the Drude model, the negative magnetoresistance peak close to $B=0 \mathrm{~T}$ has not been taken into account.

Results corresponding to the best fit are presented in Fig. 3. Several channels of conductance of both electrons and holes are needed in order to fit $\rho_{x x}$ and $\rho_{x y}$. It is obvious that one should be very careful with such results - the presence of many channels in the mobility spectrum may signify that the true mechanism behind the data is different. Nevertheless, three main contributions give a correct fit: holes $\left(\mu \approx 8000 \mathrm{~cm}^{2} /(\mathrm{Vs})\right.$, $\left.n_{\mathrm{s}}=2.0 \times 10^{11} \mathrm{~cm}^{-2}\right)$, electrons $\left(5000 \mathrm{~cm}^{2} /(\mathrm{V} \mathrm{s})\right.$, $\left.n_{\mathrm{s}}=2.0 \times 10^{11} \mathrm{~cm}^{-2}\right)$ and low mobility carriers $\left(\mu<1000 \mathrm{~cm}^{2} /(\mathrm{Vs}), n_{\mathrm{s}}>5.0 \times 10^{13} \mathrm{~cm}^{-2}\right)$. The latter most probably reflects the charge at the interface,

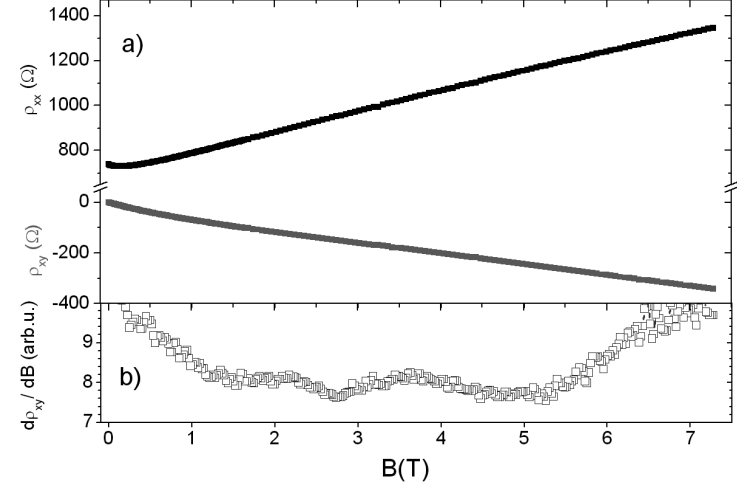

Fig. 2. (a) Resistivity tensor: $\rho_{x x}$ (black line) and $\rho_{x y}$ (grey line) measured as a function of magnetic field perpendicular to the layer, (b) $\mathrm{d} \rho_{x y} / \mathrm{d} B$ curve revealing oscillations.

which has already been observed [8] and also fairly corresponds to the linear trend of the classical Hall effect. The presence of electrons with a relatively high mobility is much more controversial because they should be visible in the quantum oscillations at high field. Therefore we performed additional measurements.
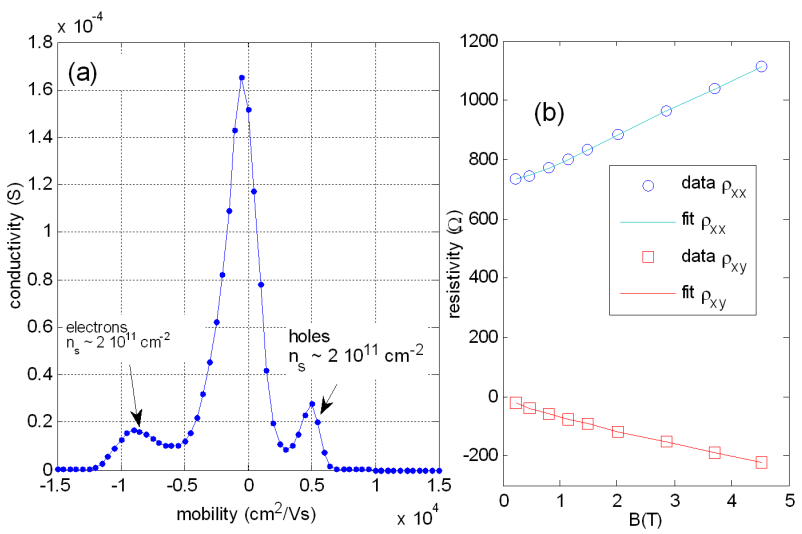

Fig. 3. Results obtained by the maximum entropy mobility spectrum [7]. In this method, no specific formulae are used to fit the resistivities. At the contrary, the fit of the resistivities gives the conductivity as a function of mobility, and then this conductivity should be compared with existing theories. (a) Partial conductivities as a function of the mobility. Negative mobility stands for electrons, positive - for holes. (b) Fit of the experimental data obtained with this method.

A small alternating magnetic field (of amplitude $1 \mathrm{mT}$ and frequency $14 \mathrm{~Hz}$ ) was added to the direct $B$ component and a derivative of $\rho_{x x}$ and $\rho_{x y}$ over magnetic field was measured. The $\mathrm{d} \rho_{x y} / \mathrm{d} B$ curve is presented in the inset to Fig. 2. A few oscillations were resolved. We attribute them to the Shubnikov-de Haas oscillations in the graphene planes. A rough estimation of the mobility can be made, knowing that the oscillations start at about $1.5 \mathrm{~T}$. The corresponding mobility equals $7000 \mathrm{~cm}^{2} /(\mathrm{V} \mathrm{s})$, 
with $n_{\mathrm{s}}=1.310^{11} \mathrm{~cm}^{-2}$. Therefore these results are in fair agreement with the mobility spectrum analysis.

\section{Conclusions}

Transport properties of epitaxial graphene layers, grown on $\mathrm{C}$ polarity were studied. The two samples have linear magnetoresistance, similarly to graphite samples. They are two-dimensional, inhomogeneous and highly disordered (with a probable weak localization peak). Several observations suggest that sample \#313 is less disordered. In this sample, the mobility is about $7000 \mathrm{~cm}^{2} /(\mathrm{V} \mathrm{s})$ for both electrons and holes. Several conduction channels have to be invoked in order to explain both the curvature and the slope of the Hall resistance as well as the observation of weak Shubnikov-de Haas oscillations.

\section{Acknowledgments}

This work was partially supported by research framework program MTKD-CT-2005-029671, by a grant of the Ministry of Science and Higher Education, No. 395/N-PICS-FR/2009/0 and by PBZ-MEiN-6/2/2006.

\section{References}

[1] W. Strupinski, R. Bozek, J. Borysiuk, K. Kosciewicz, A. Wysmolek, R. Stepniewski, J.M. Baranowski, Mater. Sci. Forum 615-617, 199 (2009).

[2] K. Grodecki, A. Drabińska, R. Bożek, A. Wysmołek, K.P. Korona, W. Strupiński, J. Borysiuk, R. Stępniewski, J.M. Baranowski, Acta Phys. Pol. A, 116, 835 (2009).

[3] G. Bergmann, Phys. Rep. 107, 1 (1984).

[4] Vadim V. Cheianov, A.P. Dmitriev, V.Yu. Kachorovskii, Phys. Rev. B 68, 201304 (2003).

[5] N.D. Brandt, S.M. Chudinov, Ya.G. Ponomarev, Modern Problems in Condensed Matter Sciences, Elsevier, Amsterdam 1988.

[6] M.M. Parish, P.B. Littlewood, Nature 38, 1544 (2003).

[7] S. Kiatgamolchai, M. Myronov, O.A. Mironov, V.G. Kantser, E.H.C. Parker, T.E. Whall, Phys. Rev. E 66, 036705 (2002).

[8] W.A. de Heer, C. Berger, X. Wu, P.N. First, E.H. Conrad, X. Li, T. Li, M. Sprinkle, J. Hass, M.L. Sadowski, M. Potemski, G. Martinez, Solid State Commun. 143, 92 (2007). 